\title{
Oncologists and COVID-19 in Indonesia: What can we learn and must do?
}

\author{
Bayu Brahma
}

Department of Surgical Oncology, Dharmais Cancer Hospital

The novel coronavirus disease (COVID-19), which is caused by severe acute respiratory syndrome coronavirus 2 (SARS-CoV-2), has spread to many countries, including Indonesia. The outbreak started within early March 2020 and in just less than a month the virus has infected 1285 patients and 114 death in Indonesia by March 30, 2020 [1]. It does not only take many lives of patients, but also our colleagues as health care providers. On behalf of the Indonesian Journal of Cancer, we would like to express our deepest condolences to all patients and especially to our doctors, nurses, and all health care workers, who could not survive in the battle against this virus.

Looking at how serious the disease is, it is estimated that the situation will give major changes to cancer patients' management, and unfortunately, it is happening in the middle of our efforts to upgrade cancer management in Indonesia. It is going to be a hard time, but we must be ready to overcome the COVID-19 crisis in the field of oncology. Let us take a brief look at published articles and recommendations in oncology.

A recent publication by Liang et al. [2] in China revealed that cancer patients with COVID-19 had higher risk of severe events, which were defined by more frequent intensive care unit admission, requirement of mechanical ventilator, and higher death rate, hence they also proposed to withhold chemotherapy and elective surgery for stable cancer in the endemic areas. However, this initial result of 18 cancer patients out of 1590 COVID-19 cases should be interpreted cautiously because of insufficient evidence to be recommended in every cancer patient [3]. More robust evidence is needed to address this issue in the field of cancer, from prevention, screening, advances in therapies, until palliative management. In the meantime, some guidelines have been proposed by several oncology socities [4-6]. Postponing cancer screening and elective surgeries such as in benign disease and risk-reducing surgery soundwise for the time being, but surgical oncologists should remember that most of the cancer surgeries cannot be considered "elective". American College of Surgeons has also released a triage guideline for surgical cases and recommended using the Elective Surgery Acuity Scale (ESAS) from St. Louis University to assist surgical decision-making [5]. To date, no direct evidence has been reported to support withholding radiotherapy, chemotherapy, and immunotherapy in daily cases, although some practice changes in several situations such as postponing, switching, or stopping aggressive adjuvant treatment in stable and low-risk cancer could be considered [4-5]. We must underline the potential harm of delaying cancer treatment and the benefit of reducing the risk of COVID-19 infection or vice versa. Individual discussions with patients should be made because many factors will contribute to giving the best answer.

We are preparing for skyrocketing COVID-19 cases in Indonesia within the next few days or weeks. How do we prepare without letting behind our main goal as oncologists to care for cancer patients? A published article by Ueda et al. [7] could be a good example for us to start with and to learn how they managed the cancer service during the early outbreak in Washington. They started with patients' triage, education for patient and family through handouts and web-site, and strengthening the policy of "stay at home". A phone triage line, providing personal protective equipment (PPE), and also test to symptomatic medical staff, were provided. In outpatient service, rescheduling visits of "well" patients, or postponing the second consultation for patients who already had treatment access in initial health care, were being conducted. Telemedicine also plays an important part of their strategy. Cancer surgery was their priority when PPE, team members, and bed capacity were available. A surgeon-to-patient phone call discussion was made when a delay in schedule was expected. Many patients with aggressive hematologic malignancies were managed not to get a treatment delay and planned to conduct a limitation for clinical trials except for the studies that will bring benefit to participants. They also mentioned that it is imperative to discuss ethical issues in the end-of-life setting when a final-stage patient acquires COVID-19 [7].

Managing cancer surgeries in our surgical oncology unit is not so simple even in the time before COVID-19, since many complex and urgent cancer cases were referred to us. When the outbreak occurred, the continuity to perform surgery has been even more challenging for us. We decided to proceed with the surgery and several adjustments were made to make 
sure the safety of patients and team members. Neither benign cases nor breast reconstructions are performed at the moment. Emergency conditons, aggressivebehavior cancer, and post-neoadjuvant chemotherapy surgeries are also prioritized. The onco-microsurgical reconstruction is only performed for complex head and neck cancer, soft tissue sarcoma, and cancer treatmentrelated lymphedema with the risk of having dermatolymphangioadenitis. Having an urgent complex case on the table, I am fortunate and honored to work with dedicated colleagues and operating room team who are willing to follow our strict rules: we do not do multiple surgeries or outpatient clinic service on the scheduled day; the surgery must be started early to avoid late hours working time; which could reduce the physical and mental health of each team member; everyone must stay focused with their parts, so "quickin-quick-out" surgery can be accomplished to prevent or reduce patients' immunosuppressive effect due to a long surgery; a well-screened patient and the availability of PPE is mandatory before the surgery is started.

It is just a modest example and we are aware that adjustment of our current protocol should be done when new scientific evidence and hospital policies are made. As the world is struggling for the battle against COVID-19, our role as oncologists, especially in Indonesia is very crucial for cancer management. Indonesia has a different situation compared to the other countries. In the current crisis, we must act scientifically and creatively. Thus, urgent works are needed: scientific COVID-19 and cancer management recommendations must be released from our oncology societies or related hospitals; hospitals' board must establish firm policies and logistics which could protect the safety of patients and medical workers; any kind of scientific study related to cancer and COVID-19 in Indonesia must be endorsed and published. It will not be easy, but once we pass the test, we will be pleased to know that we have made a significant contribution to save our patients, others, and knowledge.

\section{REFERENCES}

1. Ministry of Health Republic of Indonesia. COVID-19: Situasi kasus Indonesia [Internet]. 2020 [cited 30 March 2020]. Available from: https://infeksiemerging. kemkes.go.id/

2. Liang W, Guan W, Chen R, Wang W, Li J, Xu K, et al. Cancer patients in SARS-CoV-2 infection: A nationwide analysis in China. Lancet Oncol. 2020;21(3):335-7

3. Xia Y, Jin R, Zhao J, Li W, Shen H. Risk of COVID-19 for cancer patients. Lancet Oncol. 2020;2045(20):30150.

4. American Society of Clinical Oncology. COVID-19 clinical oncology frequently asked questions (FAQs) [Internet]. 2020 [cited 28 March 2020]. Available from: https://www.asco.org/sites/new-www.asco. org/files/content-files/blog-release/pdf/COVID-19Clinical\%200ncology-FAQs-3-12-2020.pdf

5. American College of Surgeons. COVID-19: COVID-19: Guidance for triage of non-emergent surgical procedures [internet]. 2020 [cited 28 March 2020]. Available from: https://www.facs.org/covid-19/ clinical-guidance/triage

6. The European Society of Surgical Oncology. ESSO statement on COVID-19 [Internet]. 2020 [cited 28 March 2020]. Available from: https://www.essoweb. org/news/esso-statement-covid-19/

7. Ueda M, Martins R, Hendrie PC, McDonnell T, Crews $J R$, Wong $T L$, et al. Managing cancer care during the COVID-19 pandemic: Agility and collaboration toward a common goal. JNCCN-Journal Natl Compr Cancer Netw. 2020;18(4):1-4.

Corresponding author:

Bayu Brahma, MD

Editor-in-Chief

Indonesian Journal of Cancer

Email: bbrahma_s@yahoo.com 